\section{P 113 MANAGEMENT OF METASTATIC SPINAL CORD COMPRESSION: AUDIT}

Sarika Hanchanale, Karen Neoh, Joy Waldock, James Lenton, Jay Naik. Pinderfields General Hospital, Midyorkshire Hospitals NHS trust, Leeds, UK

10.1136/bmjspcare-2014-000654.154

Introduction Metastatic spinal cord compression (MSCC) is a medical emergency, prompt management is required to preserve neurological function. A retrospective audit of MSCC management in the Mid Yorkshire NHS trust in 2012 was conducted.

Aims To compare current practice to NICE guidance, and also record patients' diagnosis, signs, symptoms and three month survival. MRI and radiotherapy time from request were calculated. The prescription of dexamethasone, Low molecular weight heparin (LMWH), analgesia and gastric protection were assessed.

Results Twenty seven patients were identified as having MSCC. The most common cancers were lung, prostate and breast, but $15 \%$ of patients had no malignant diagnosis at the time of presentation. Most patients had back pain (23), with duration from one day to three months. Fifteen patients had reduced mobility and four patients had bladder dysfunction. Twenty three patients were suspected of having MSCC, three were suspected of having spinal metastasis and one patient was thought to have a slipped disc. Only 53\% of the patients with suspected MSCC had an MRI performed within 24 hours. The regional oncology team was contacted in all cases. LMWH and analgesia were given in $96 \%$ of the patients. All patients received steroids, but dexamethasone prescribing and dose reduction was inconsistent. Two thirds of patients had radiotherapy within one day. Three month survival was $56 \%$.

Conclusion MRI scan within 24 hours was just over 50\% and should be $100 \%$; reasons for delay include patients being too unwell to transfer and transport issues, but these were only in a few cases. This is a complex problem, especially if patients are out patients, and requires improved radiology capacity. We recommended staff education and a local protocol, which should include guidelines on standardisation of dexamethasone prescribing. The audit should be repeated once the protocol has been in place for 6-12 months. 